\title{
Single ventricle, many arrhythmias
}

\author{
Robert Whitehill ${ }^{1,2}$, Peter Fischbach ${ }^{1,2}$, Matthew E. Oster ${ }^{1,2}$ \\ ${ }^{1}$ Department of Pediatrics, Emory University School of Medicine, Atlanta, GA, USA; ${ }^{2}$ Division of Cardiology, Children's Healthcare of Atlanta, \\ Atlanta, GA, USA \\ Correspondence to: Matthew Oster, MD, MPH. Children's Healthcare of Atlanta, 2835 Brandywine Rd, Suite 400, Atlanta, GA 30341 , USA. \\ Email: osterm@kidsheart.com. \\ Provenance: This is an invited Editorial commissioned by Executive Editor-in-Chief Jianxing He (Director of the Thoracic Surgery Department, The \\ First Affiliated Hospital of Guangzhou Medical University, Guangzhou, China). \\ Comment on: Hall EJ, Smith AH, Fish FA, et al. Association of Shunt Type With Arrhythmias After Norwood Procedure. Ann Thorac Surg \\ 2018;105:629-36.
}

Submitted Sep 06, 2018. Accepted for publication Sep 11, 2018.

doi: $10.21037 /$ jtd.2018.09.66

View this article at: http://dx.doi.org/10.21037/jtd.2018.09.66

As the wheels of progress churn forward in cardiovascular care and cardiac surgical technique, the survival rate for infants born with single ventricle physiology, once a universally fatal disease, has improved dramatically. The single ventricle reconstruction (SVR) trial recently reported an overall survival in these patients of $62 \%$ at 6 years of follow up $(1,2)$. Fontan's original operation featuring an atriopulmonary connection combined with an end-to-end SVC to PA connection for tricuspid atresia was a breakthrough and created normal or near normal systemic saturations in single ventricle patients. However, the abnormal physiology inherent to this circulation leads to progressive dilation of the systemic venous atrium and frequent arrhythmic complications. Modifications of the original Fontan operation were also instituted to not only address survival but also to improve morbidities that became apparent in the short, medium and long term follow up associated with the hemodynamic consequences of the original approach. The introduction of the lateral tunnel Fontan was designed to improve the energy loss of the venous system through the systemic and pulmonary circulations while also decreasing the burden of atrial arrhythmias. This technique was further refined to the extracardiac Fontan, though this strategy is frequently limited by the need to balance the size of the extracardiac conduit and the patient (3). Similarly, the surgical approach to the initial Stage I palliation has evolved over time. The initial (and still frequently used) palliation utilized a modified Blalock-Taussig shunt (MBTS) to provide a stable source of pulmonary blood in addition to the reconstruction of the aortic arch. Sano et al. revised this by incorporating a right ventricle to pulmonary artery shunt (RVPAS) and eliminating the MBTS. One of the motivations behind this development was to eliminate potential coronary steal which many thought played a role in a sizable percentage of interstage mortality. Many centers have adopted this technique and the SVR trial run by the Pediatric Heart Network was undertaken to examine the short and medium term outcomes after each type of shunt. This multicenter 6-year follow up study showed no statistically significant difference in transplant-free survival based on type of shunt at the Stage I procedure. With these improvements in survival and changes in surgical technique, providers are now able to address the significant morbidity of this vulnerable population. One major post-operative morbidity remains electrophysiologic abnormalities including tachyarrhythmias, conduction disturbances and bradyarrhythmia.

Many groups have studied the incidence of arrhythmias following single ventricle palliation. Pediatric and adult studies have all reported a high cumulative incidence, with supraventricular arrhythmias being described most often. As mentioned previously, the Fontan procedure has been modified in an attempt to reduce electrophysiologic morbidity during the final stage of palliation. While atrial arrhythmias are frequent, the incidence of ventricular arrhythmia (VA) is much less. One long term follow up of patients following a Fontan procedure reported an 
incidence of ventricular tachycardia of $5 \%$ compared to $35 \%$ with atrial flutter, $19 \%$ with atrial fibrillation and $13 \%$ with other atrial tachycardias (4). Pediatric studies have also been published focusing on the interstage period between the Norwood and Glen with a reported incidence of ventricular tachycardia of $1-4 \%(5-8)$. With the increased incorporation of the RVPAS during the initial palliation, it has been hypothesized that the incidence of VA may increase.

The RVPAS theoretically provides the ideal substrate for VA. A ventriculotomy is made in a compromised ventricle with implantation of the shunt in the right ventricular outflow tract. As the shunt provides a central obstruction and the surrounding, scarred myocardium the substrate for slow conduction, a circuit for ventricular macroreentry is created. The physiology of this circuit mimics that which is present in patients with repaired Tetralogy of Fallot who have a known risk for VA (9). The electrophysiologic properties of Tetralogy patients have been well studied and VA a known complication with an incidence of ventricular tachycardia in patients with Tetralogy of Fallot published as high as $14.6 \%$ in one multicenter study with long term follow up (10). While the RV-to-PA conduit is taken down at the time of the Glenn in the single ventricle staged palliation, the resulting scar and fibrosis remain, leaving the potential for similar issues in the long term. As the field continues to evolve, study of the long term incidence of VA is certainly warranted.

In this volume of the Fournal of Thoracic Disease, the authors report results from a single institution with the objective of assessing differences in arrhythmia burden between shunt types in patients with single right ventricular physiology. The publication represents another contribution to this growing body of literature regarding rhythm problems after single ventricle palliation and their impact on prognosis (11). One interesting observation was that the incidence of VA in their cohort was greater than that which has been reported elsewhere in this population. They conclude that VAs were indeed higher in the RVPAS group and note that the majority occurred in the acute period after the Norwood. In the RVPAS group, 29\% developed a VA compared with $14 \%$ in the BTS group, a finding which lends some credence to the theory that the RVPAS increases the risk of VAs in the acute, postoperative period. As for the type of VA, only 1 patient had polymorphic ventricular tachycardia (VT) in their cohort while the remainder of VAs were monomorphic. Further description of the VAs was not available and is an area for future study to determine if these were macroreentry. Interestingly, this level of VA has not been reported in several other studies examining rhythm problems in this population. The increased incidence of overall VAs found in the study is significant and is likely related to the prospective nature of the data collection compared to the retrospective method of the other publications. Once more, their definitions of VA may have increased the yield since they incorporated accelerated idioventricular rhythms in additional to VT. While accelerated VA can be hemodynamically significant given the loss of atrial and ventricular synchrony, it makes a direct comparison to the incidence of VA in other studies difficult. Other variables for consideration in the incidence of VA in this study include the specific patient population, different surgical techniques, medications, monitoring and a myriad of other differences in patient management between centers. Shifting focus to the clinical implications of the reported VAs, the authors report that most were transient and did not require treatment, though 4 episodes of accelerated ventricular rhythm and 2 episodes of monomorphic VT did. No patients in this cohort died from a documented VA though one patient had a cardiac arrest due to a junctional tachycardia. The data from this study would suggest that while the RVPAS was indeed associated with a higher incidence of VAs, many did not require treatment. This raises questions about the significance of these rhythms in the long term, however. The study was not able to show a difference in survival on multivariate analysis by presence of VA though they report a number of the deaths in their cohort had an unknown etiology. Similar rates of death reported due to an unknown cause have been reported elsewhere and have led some to hypothesize that occult arrhythmia after hospital discharge may be the culprit. More research is needed in this area and exploration of outpatient monitoring may be warranted to further define the impact VAs have on survival. Regardless, this study seems to conclude that with prospective methods and broad definitions, RVPAS patients were shown to have more acute VAs than patients undergoing Norwood with a MBTS. The clinical significance in the acute phase appeared to be minimal as most did not require treatment and none were a direct, documented cause of mortality.

As this group of patients ages it will hopefully become clearer whether the shunt type has a discernible difference in the incidence of arrhythmias, and VAs in particular. Hall et al.'s patients had a median follow up of 773 days which is still relatively short. Arrhythmia remains a common source of complications in single, right ventricle physiology 
and is the product of numerous, complex factors in the acute and long term. The authors report a high incidence of all arrhythmias and VAs using a prospective method of data collection and broader definitions than have been published previously. This unique approach helps shed light on the concern regarding RVPAS and a perceived increased risk for VA. However, this study also showed that most of these episodes did not require intervention, which lessens the clinical significance of the previous finding in the short term. Regardless, this paper and others have set the stage for long term follow up as this population ages and the hemodynamic and arrhythmogenic potential of the various shunt types is realized. Only time will tell what type of footprint the types of shunt will have on the distant morbidity and mortality of this fragile population.

\section{Acknowledgements}

None.

\section{Footnote}

Conflicts of Interest: The authors have no conflicts of interest to declare.

\section{References}

1. Newburger JW, Sleeper LA, Gaynor JW, et al. TransplantFree Survival and Interventions at 6 Years in the SVR Trial. Circulation 2018;137:2246-53.

2. Ohye RG, Schonbeck JV, Eghtesady P, et al. Cause, timing, and location of death in the Single Ventricle Reconstruction trial. J Thorac Cardiovasc Surg

Cite this article as: Whitehill R, Fischbach P, Oster ME. Single ventricle, many arrhythmias. J Thorac Dis 2018;10(Suppl 33):S4040-S4042. doi: 10.21037/jtd.2018.09.66
2012;144:907-14.

3. d'Udekem Y, Iyengar AJ, Cochrane AD, et al. The Fontan procedure: contemporary techniques have improved longterm outcomes. Circulation 2007;116:I157-64.

4. Pundi KN, Pundi KN, Johnson JN, et al. Sudden cardiac death and late arrhythmias after the Fontan operation. Congenit Heart Dis 2017;12:17-23.

5. Oster ME, Chen S, Dagincourt N, et al. Development and impact of arrhythmias after the Norwood procedure: A report from the Pediatric Heart Network. J Thorac Cardiovasc Surg 2017;153:638-45.e2.

6. McFerson MC, McCanta AC, Pan Z, et al. Tachyarrhythmias after the Norwood procedure: relationship and effect of vasoactive agents. Pediatr Cardiol 2014;35:668-75.

7. Trivedi B, Smith PB, Barker PC, et al. Arrhythmias in patients with hypoplastic left heart syndrome. Am Heart J 2011;161:138-44.

8. Gist KM, Schuchardt EL, Moroze MK, et al. Tachyarrhythmia following Norwood operation: a singlecenter experience. World J Pediatr Congenit Heart Surg 2014;5:206-10.

9. Kapel GF, Sacher F, Dekkers OM, et al. Arrhythmogenic anatomical isthmuses identified by electroanatomical mapping are the substrate for ventricular tachycardia in repaired Tetralogy of Fallot. Eur Heart J 2017;38:268-76.

10. Khairy P, Aboulhosn J, Gurvitz MZ, et al. Arrhythmia burden in adults with surgically repaired tetralogy of Fallot: a multi-institutional study. Circulation 2010;122:868-75.

11. Hall EJ, Smith AH, Fish FA, et al. Association of Shunt Type With Arrhythmias After Norwood Procedure. Ann Thorac Surg 2018;105:629-36. 